\title{
A Building Energy-Saving Method of Small High-Rise Office Building Based on BIM Model
}

\author{
Junqing Li $\mathbb{D},{ }^{1}$ Yongbing $\mathrm{Li} \mathbb{D},{ }^{2}$ and Xuan Zhang $\mathbb{D}^{1}$ \\ ${ }^{1}$ School of Engineering Technology, Hulunbuir University, Inner Mongolia, Hulun Buir 021008, China \\ ${ }^{2}$ School of Engineering Management, Inner Mongolia University of Finance and Economics, Inner Mongolia, \\ Hohhot 010051, China \\ Correspondence should be addressed to Junqing Li; lijunqing@hlbec.edu.cn
}

Received 27 December 2021; Accepted 12 January 2022; Published 31 January 2022

Academic Editor: Sikandar Ali

Copyright (C) 2022 Junqing Li et al. This is an open access article distributed under the Creative Commons Attribution License, which permits unrestricted use, distribution, and reproduction in any medium, provided the original work is properly cited.

Building green energy-saving is an important area of current architectural application research. Artificial intelligence algorithm can effectively improve the design of building energy-saving digital, intelligent level. Therefore, this paper proposes the use of BIM model based on genetic algorithms to optimize the application of building energy conservation research. In this paper, the basic structure of genetic algorithm and advantages and disadvantages of the algorithm are described. According to the need of optimal design of building energy-saving integration, multiobjective optimization function is introduced and a multiobjective optimization genetic algorithm is established to improve the prediction effect of building energy efficiency model. Finally, results of analysis of some of the influencing factors and the simulation test of building energy-saving integrated optimization algorithm in small high-rise office building show that the improved genetic algorithm can effectively improve the effect of energy-saving integrated optimization and has good application prospects.

\section{Introduction}

China is the second largest economy in the world today and the largest developing country in the world. In the long-term growth stage, the domestic urban construction department steadily pushed forward the urbanization and urbanization construction, resulting in a substantial increase in the urbanrural construction area [1-3]. China is also a large energy consumer and the total energy consumption of buildings has remained high for a long time. Compared with developed countries such as Europe and the United States, there is still a big gap. In the background of the national gross floor area and the continuous development of the construction industry, the energy consumption proportion in building energy is facing a new test for the energy situation $[2,4]$. At present, energy-saving design of domestic buildings is the focus of attention. Due to the vast territory of our country, large changes in environmental climate, thermal performance of buildings, equipment efficiency, and other factors, it is still very difficult to build an organic symbiotic architecture with advanced design concepts $[2,5]$. One of the main shortcomings is that the concept of overall optimization has not been established in the design of building energy efficiency, and all aspects of building functional design are in their own independent state. The evaluation of the effect of building energy-saving technologies also stops counting the number of building energy-saving technologies and considers energy-saving construction as the accumulation of energy-saving technologies $[1,6]$. As a result, building energy efficiency applications always come with a situation where the overall energy saving function and the energy efficiency index are not satisfactory $[1,7]$. Therefore, based on the previous single-factor analysis, several genetic algorithms-based BIM model is proposed in this paper to conduct a comprehensive study of building energy efficiency after considering the respective scattered factors comprehensively. The BIM model of a small high-rise office building is shown in Figure 1. 


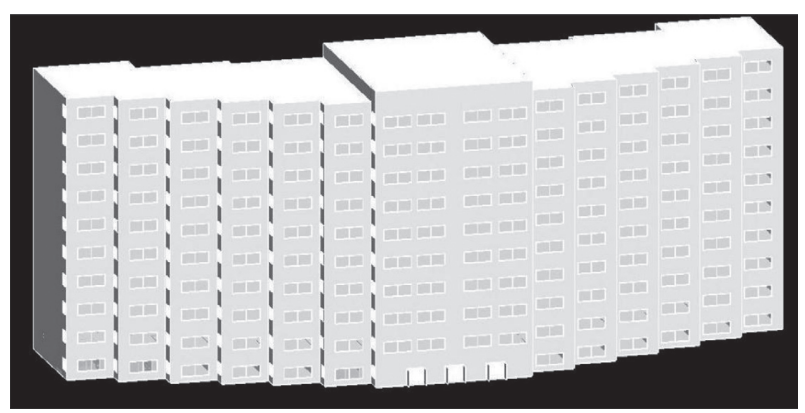

FIgURE 1: BIM model of a small high-rise office building.

\section{State of the Art}

Genetic algorithm has solved the problem, to determine the coding scheme, fitness function and genetic operators, and other parameters, to carry out independent organization search. Genetic algorithms, such as the optimal value of the global random search and building energy efficiency integration optimization, are involved in the energy-saving goals, energy-saving conditions, energy-saving parameters, and many other mathematical factors with nonlinear, multiobjective, and other requirements to adapt, so it is essential to use genetic algorithms for energy-efficient design. There is a good rationale for optimizing research [8]. The prototype of genetic algorithm is the study of natural and artificial adaptive system put forward by Professor Holland in the 1960s. The United States is the first in the world to innovate an adaptive optimization genetic algorithm that can effectively solve complex systems and complicated computations [9]. Afterwards, scholars carried out a large number of pure numerical optimization experiments using genetic algorithms and formed the basic structure of genetic algorithm in the 1980s, laying an important foundation for subsequent genetic algorithms [10]. The genetic algorithm uses multiple iterations to find new approximate solutions in the process of continuous selection, crossover, and optimization according to the fitness values generated by individuals in different problem domains and simulates the process of population evolution in the natural environment so that the obtained new individuals are better able to adapt to environmental requirements than past ones. Genetic factors can improve and optimize population assemblages continuously, keeping excellent structures to the next generation or generating better structures [11]. In the search to take the choice, crossover, mutation, and other operations, get better nextgeneration samples, allowing a new generation of populations always maintain the optimal structure [12].

With the rapid development of Internet information technology hardware and software, the construction industry has undergone tremendous changes from design to construction under the support of computer-aided technology. The direction of the most advanced changes is not only the construction process of building materials but also focuses more on the life cycle of building construction and provides a more efficient and convenient management and uses platform for building design and construction in digital form through various virtual expressions of building information before the building entity is completed. Building information modeling (BIM) is a brand-new construction management model introduced in China at the beginning of the new century. Its design core is a three-dimensional building model, which mainly uses computer information system to analyze the physical, functional, and other characteristics of the process of digital expression. As a rich and complete database, BIM is an intelligent and parametric simulation system for building objects, which can help to optimize the design of energy efficient buildings to a more advanced development path.

\section{Methodology}

3.1. Genetic Algorithm. Genetic algorithm embodies the evolving wisdom achievements of human society and is an important manifestation of the natural law of "the survival of the fittest and the discomfort being eliminated." The main theoretical basis is the use of mathematical models to simulate the process of biological evolution. The genetic algorithm brings the process of selecting the optimal solution into the process of biological evolution, taking the sample population as the representative of the problem and the genetic factors into the sample population. After choosing, crossing, mutation, and other operations, a new generation of population is obtained, the optimizing will be iteratively operated, and the individual with the maximum fitness is finally selected as the optimal solution. Simple genetic algorithm (SGA) is an important basic algorithm for improving genetic algorithms. It generally includes the main processes of coding, establishing initial population, evaluating individual fitness, and choosing design genetic operators. The basic steps of genetic algorithm are shown in Figure 2.

In the design of genetic operators, we need to finally set the reasonable range and size of the genetic algorithm parameters through multiple experiments. This article describes the calculation of operator selection using roulette. The probability of each individual entering the next-generation population is determined by the ratio of individual fitness to the sum of individual fitness in the entire population. The greater the individual fitness value, the higher the probability of being selected and entering the next generation. Let the population size be $M$, the fitness value of individual $i$ be denoted by $f_{1}$, and the probability that the individual is selected will be calculated by formula (1). The calculation process is to first calculate each fitness value of population individuals $f_{1}, i=1,2, \ldots, M$;

$$
P_{i}=\frac{f_{i}}{\sum_{i=1}^{M} f_{i}} .
$$

The fitness of all individuals in the population is calculated using the following equation:

$$
F=\sum_{i=1}^{M} f_{i} .
$$

The probability of choosing individuals is calculated using the following formula: 


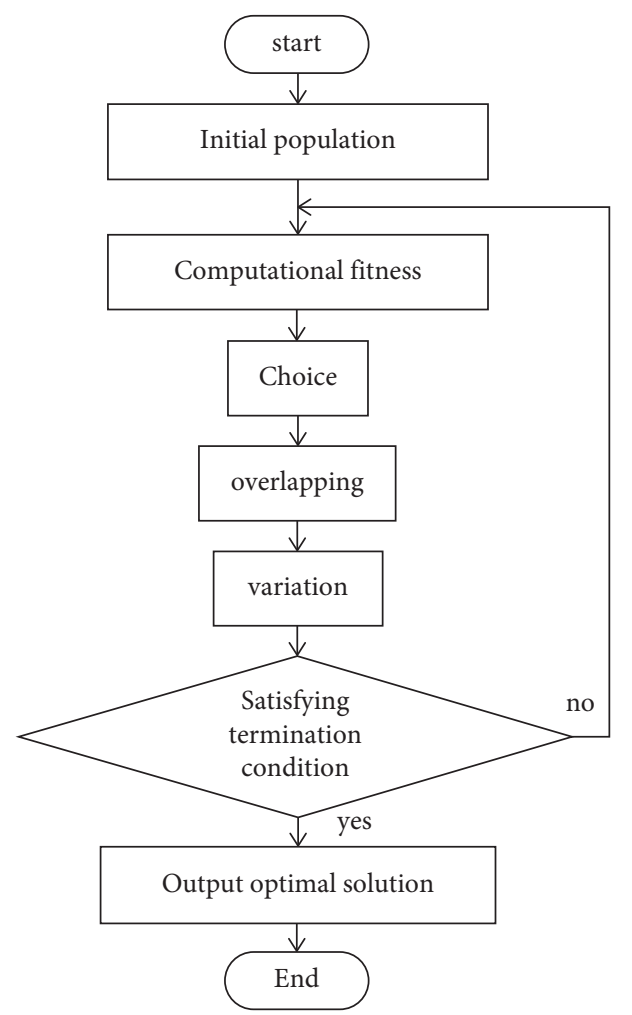

Figure 2: Operation diagram of genetic algorithm.

$$
P_{k}=\frac{f_{k}}{\sum_{i=1}^{M} f_{i}}, k=1,2, \ldots M
$$

The above steps are runner method. The selection process is to choose the M-turn process. Each time the following method is also selected, a new individual is added to the new population. Set the pseudorandom number $r$ as $[0,1]$ range evenly distributed. At $r \leq q_{1}$, the first individual is selected; otherwise, the $k$ th individual $(2 \leq k \leq M)$ is selected, and the setting of $q_{k-1} \leq r \leq q_{k}$ is established.

In the basic chromosome encoding, the genetic algorithm uses a binary encoding, the general use of fixed-length binary symbols represents different individuals of the population, and its corresponding gene also consists of a symbol set $\{0,1\}$. Genetic algorithm of the individual tree structure encoding is shown in Figure 3.

In the initial population selection, the genetic algorithm will randomly select the initial population of the object at the beginning; select the value of 20 to 100 . In the constrained optimization problem, the initial population should be based on the diversity, randomness, and evenness to ensure the feasibility of individual population. Under this requirement, it is very difficult to reach the target by randomly selecting the population. In the case of many dimensions and constraints in many cases, applying only the stochastic algorithm will prolong the selection time of the initial population and directly affect the efficiency of the genetic algorithm. Therefore, this paper proposes to study two aspects of the initial population generation. One is that the initial population is generated by means of internal modification based

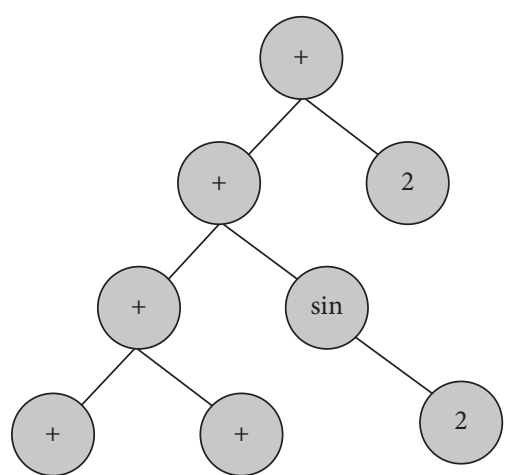

FIGURE 3: Individual tree structure coding graph of genetic algorithm.

on the points in the feasible field. Second, in the case that man cannot give an initial point, scientific search is used to find a point. The searching process for the initial population is shown in Figure 4. Here, $x_{1}^{(1)}$ is the point inside the feasible region, which is the initial interior point of the request. After the initial population finishes searching for the first individual after $x_{1}$, a subsequent individual $x_{2}=\left[x_{1}, x_{1}, \ldots, x_{n}\right]$ is randomly generated. If $x_{2}$ is a viable individual, proceed to the next individual, $x_{3}$. If $x_{2}$ is not a viable individual, adjust it according to $x_{2} \Leftarrow x_{1}+\alpha\left(x_{1}+x_{2}\right)$. Here is the $\alpha$ contraction factor, the value of the choice between [0,1]. At $\alpha=$ 0.5 , the point at which new points are available is between $x_{1}$ and $x_{2}$. Then, follow the same method to find all the points in the initial population that are needed.

3.2. Optimization Genetic Algorithm. Although genetic algorithm has the advantages of other algorithms do not have, there are some shortcomings. The genetic algorithm adopts the method of fixed strategy parameters, the optimization results are not good, and there is no way to solve the problem of the change of strategy parameters and the dynamic in the genetic evolution, which is highlighted in the situation that the crossover probability and the mutation probability cannot be controlled [13]. When applying genetic algorithms in different fields, we need to combine the professional needs with the basic genetic algorithms according to the characteristics of each specialty and update and improve the genetic algorithms effectively to help the genetic algorithm to be effective under different types of problems. In order to solve the problem of multiobjective solution in building optimization, aiming at the design requirements in the field of building energy conservation, this paper aims at solving the conflicting and incomparable subfunctions that may appear in the optimization objective function, finding the global optimal solution in the range of feasible solutions. We can only find out the optimal solution set of the objective optimization. Introducing the optimal solution set is not only the concept of Pareto optimal solution. Pareto optimal solution is a resource allocation strategy; the principle is that in the case of which party did not let worse, at least let a party to move in the direction of development; it is commonly 


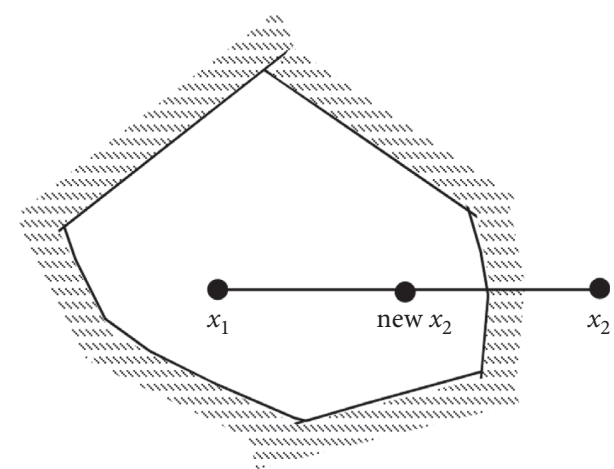

FIGURE 4: The relationship between the new individual and the original individual.

used in multiobjective optimization of the main methods. The expression of the equation is as shown in equation (4), where $f(x)$ is a multiobjective optimization problem and $x^{*}$ is the optimal solution. Among them, $f: \Omega \longrightarrow R^{r}$, where $\Omega$ is a feasible solution set that satisfies the constraint and also a decision variable space that satisfies formula (5). When the objective function $f(x)$ is mapped to the set $\prod \subseteq R^{r}, \prod$ is the objective function space.

$$
\begin{aligned}
f\left(X^{*}\right)= & \text { opt } f(x) x \in \Omega(3-10), \\
\Omega= & \left\{X \in R^{n} \mid g_{i}(x) \geq 0, h_{i}(x) \geq 0\right. \\
& (i=1,2, \ldots, k ; i=1,2, \ldots, l)\} .
\end{aligned}
$$

The Pareto optimal solution set generally appears above the boundary line or plane of the objective function space. This boundary is the optimal boundary and is the tangent point of the objective function, as shown in Figure 4. The Pareto optimal boundary space changes according to the number of objective functions. When the objective function is 2 , the free boundary is linear. When the objective function is 3, the optimal boundary is surface and the objective function is 3 or more. The superior boundary is the hypersurface state. It can be seen from Figure 5 that the optimal space of the two objective functions is the solid line in the figure, which is the Pareto optimal boundary. Among them, points 1-6 are the optimal solution, and points 7-12 do not appear on the optimal boundary, so it is not the optimal solution.

The traditional multiobjective optimization is to couple each subobjective function into a total objective function and use the coupling parameters in the total objective function to carry out adaptive calculation under the optimization algorithm to get the Pareto optimal solution set. The general method is the global rule method, the weighted method, and the level of optimization method. This paper mainly combines the genetic algorithm and Pareto optimal solution set to solve the problem that the traditional algorithm is insensitive to the Pareto optimal solution set and the algorithm and knowledge in the problem field cannot be well combined. NSGA-II algorithm is one of the multiobjective genetic algorithms and is an emerging algorithm based on Pareto optimal concept selection strategy, which was proposed by Srinivas and Deb only in 2002. The main

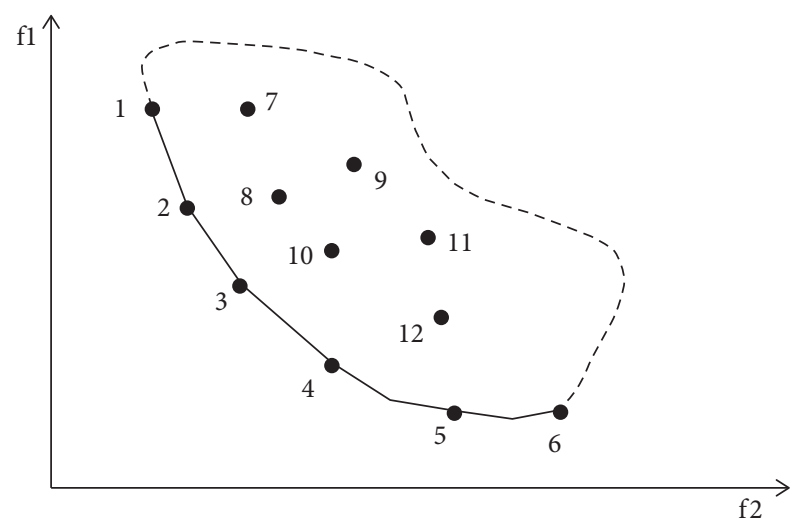

Figure 5: Pareto Optimal boundary.

flow of the algorithm is that, firstly, the population is divided into different levels according to the interrelationship among the individuals of the population, the first layer is the nondominant individual in the population, and the remaining individuals belong to the new population. The second layer includes all new populations of nondominant individuals, the rest of the formation of new populations, and the same cycle operation. In the selection process, the selection mechanism is to select the individuals in the highlevel hierarchy according to the selected strategy to achieve the scale requirement of the next-generation population. Algorithm is mainly used in the second cycle to calculate each individual $n_{i}$ and $s_{i}$, where $n_{i}$ is the number of dominant individuals and $s_{i}$ is a collection of individual dominance. According to equation $P_{k}=\left\{i \mid n_{i}-k+1=0\right\}$ to solve the nondominated and dominant set, here $P_{k}$ is a nondominant set; the rest is the dominant set. It is at $n_{i}=0$ that individual $i$ is a nondominant individual. Figure 6 is a multiobjective genetic algorithm flow.

\section{Result Analysis and Discussion}

This article focuses on building programs in the early design to reduce energy demand, in order to achieve the building energy-saving application goals. The objective of using the optimized multiobjective genetic algorithm is to find the optimal solution set that satisfies the building's construction scheme with lower energy consumption, better natural day lighting rate, and higher natural similarity. Here is a comparison of simulation testing methods to verify after the mathematical changes in temperature and frequency capacity, the natural pressure $\mathrm{Pa}$ time, the average lighting coefficient and other objective function, the optimal solution set to find, and the optimal solution. The convergence of the corresponding objective function value forms the optimal boundary of Pareto. Before the test, we first set the object of the integrated optimization of the building scheme. The object here is a rectangular office area in a small high-rise office building. The building has six sides, four sides numbered $1,2,3$, and 4 , a top number of 5 , a floor number of 6 , and openings 1 and 2 on face 1 and face 3 , respectively. The length of the building is $a$, the width is $b$, the height is $h$, the 


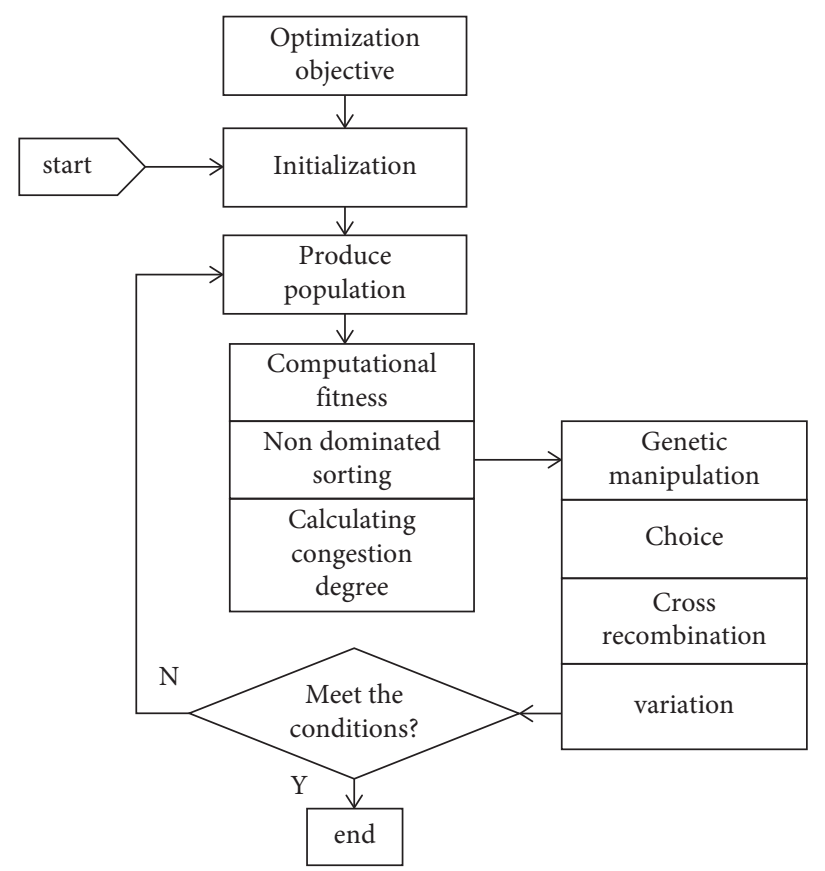

FIGURE 6: Multiobjective genetic algorithm flow.

TABLE 1: Optimization parameters and constraint parameters of building objects.

\begin{tabular}{|c|c|c|c|c|}
\hline \multirow{2}{*}{ Serial number } & \multirow{2}{*}{ Parameter name optimization } & \multirow{2}{*}{ Company } & \multicolumn{2}{|c|}{ Constraint condition } \\
\hline & & & Guangzhou & Beijing \\
\hline 1 & Orientation & $\circ$ & \multicolumn{2}{|c|}{$0-260$} \\
\hline 2 & Building chief a & M & \multicolumn{2}{|c|}{$\mathrm{a} * b=300$} \\
\hline 3 & Building width $b$ & M & \multicolumn{2}{|c|}{$1 / 2<a / b<1$} \\
\hline 4 & AG1 opening 1 area & $m^{2}$ & $\begin{array}{c}\mathrm{AG} 1 / a * h<0.4 \\
2<\mathrm{AG} 1<20\end{array}$ & $\begin{array}{c}\mathrm{AG} 1 / a * h<0.5 \\
2<\mathrm{AG} 1<20\end{array}$ \\
\hline 5 & AG2 opening 2 area & $m^{2}$ & $\begin{array}{c}\mathrm{AG} 2 / a * h<0.4 \\
2<\mathrm{AG} 2<20\end{array}$ & $\begin{array}{c}\mathrm{AG} 2 / a * h<0.3 \\
2 \leq \mathrm{AG} 2 \leq 20\end{array}$ \\
\hline 6 & Wall heat transfer coefficient $\mathrm{K} 1$ & $W /\left(m^{2} * k\right)$ & $0.7<k 1<2.5$ & $0.7 \leq k \leq 0.45$ \\
\hline 7 & Roof heat transfer coefficient $\mathrm{K} 2$ & $W /\left(m^{2} * k\right)$ & $0.4<k 5 \leq 0.9$ & $0.5 \leq k 5 \leq 1.0$ \\
\hline
\end{tabular}

area of the opening 1 is AG1, the area of the opening 2 is AG2, and the heat transfer coefficients of the wall and the roof are, respectively, $\mathrm{K} 1$ and $\mathrm{K} 2$. Here, the optimization of building objects and constraint parameters is shown in Table 1.

Using BIM model to retrieve building content database and design building energy-saving integrated optimization design system, the building solutions and energy-saving objects with different climate characteristics in Guangzhou and Beijing were selected for comprehensive design and optimization, so as to verify the effectiveness of the optimized solutions. The selected relevant parameters are shown in Table 2.

The optimal design conditions for the selected real-world building objects and the BIM model multiobjective genetic


0.43 , and pdph $(h)=6438$. The corresponding values of filtered out are BIN $\left(\mathrm{kW} h / \mathrm{m}^{2}\right)=94, \operatorname{ADF}(\%)=0.55$, and $\mathrm{PDPH}(h)=8743$, and the corresponding comparison of $\mathrm{ADF}, \mathrm{PDPH}$, and BIN is shown in Figure 7. It can be seen from the figure that the building energy consumption of the high-rise office building obtained by the method of temperature and frequency at the building energy consumption is $109 \mathrm{kw} \mathrm{h} / \mathrm{m}^{2}$. The optimal solution set of all the construction solutions is produced by the multiobjective genetic algorithm of BIM model. The building energy consumption

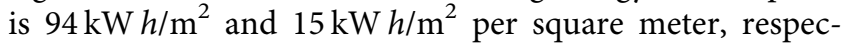
tively. The average day lighting factor of the high-rise office building is 0.43 , and the average coefficient of multiobjective genetic algorithm for BIM model is 0.55 , compared with the day lighting coefficient increased by about 29\%; natural ventilation parameters show that the natural pressure $\mathrm{Pa}$ residential hours is 6476 and BIM model multiobjective genetic algorithm is designed to produce all the optimal solutions in the construction of the natural pressure $\mathrm{Pa}$. The number is 8871 , a $37 \%$ increase compared to the better natural ventilation potential. It can be seen that the research on energy saving of small high-rise buildings based on multiobjective genetic algorithm using BIM model is successful. 
TABLE 2: Input parameter list.

\begin{tabular}{|c|c|c|c|}
\hline City & & Guangzhou & Beijing \\
\hline Dimension & & 23.2 & 39.8 \\
\hline Thermal zoning & & Hot summer and warm winter & Cold \\
\hline Built-up area & & 200 & \\
\hline Building height & & 3.5 & \\
\hline Number of people in buildings & & 10 & \\
\hline Computational algebra & & 2000 & \\
\hline Population size & & 100 & \\
\hline Crossover rate & & $0.6-1.00$ & \\
\hline Mutation rate & & $0.005-0.01$ & \\
\hline \multirow{5}{*}{$\begin{array}{l}\text { Energy consumption calculation, indoor design } \\
\text { parameters in refrigeration period }\end{array}$} & Humidity & 26 & 26 \\
\hline & Relative humidity & $50 \%$ & $50 \%$ \\
\hline & Moisture content & 10.621 & 10.621 \\
\hline & Air change times & 1 & 1 \\
\hline & Date & $4.15-11.15$ & $7.15-8.31$ \\
\hline \multirow{5}{*}{$\begin{array}{l}\text { Energy consumption calculation, indoor } \\
\text { parameter design during heating period }\end{array}$} & Humidity & 16 & 18 \\
\hline & Relative humidity & $50 \%$ & $50 \%$ \\
\hline & Moisture content & 5.6783 & 6.4987 \\
\hline & Air change times & 1 & 0.5 \\
\hline & Date & $1.15-2.15$ & $11.15-3.15$ \\
\hline Peak heat load temperature & & 5 & -16 \\
\hline Peak cold load temperature & & 35 & 36 \\
\hline Intermediate heat load temperature & & 4 & 5 \\
\hline Intermediate cooling load temperature & & 22 & 22 \\
\hline
\end{tabular}


Figure 7: Comparison of evaluation index data.

\section{Conclusion}

Genetic algorithm can simulate biological evolution and has genetic advantages so that certain goals need to be solved to get the best solution and is one of the human intelligent algorithm models. Therefore, this paper proposes the application of building energy conservation based on genetic algorithms. This article elaborates the operation and advantage of genetic algorithm and then optimizes and improves the genetic algorithm. Aiming at the problems that may appear in the optimization and application of building energy efficiency integration, a multiobjective genetic algorithm is proposed to analyze the principle and implementation of multiobjective optimal solution set. The multiobjective genetic algorithm is used to select the optimal solution in the optimal solution set. Afterwards, a multiobjective genetic algorithm is used to establish a problem model of integrated energy-saving building, the relevant constraint parameters are collected as input data, and the BIM model is used to construct the program simulation process and compares the situation of building energy efficiency index under the integrated optimization and the real state and discusses the advantages of the multiobjective genetic algorithm and draws the result of this study. The results show that the multiobjective genetic algorithm based on BIM model has good practical value for the application of building energy saving in small high-rise office buildings and can provide better intelligent solutions for reducing the overall energy consumption of buildings. However, it is also found in the research that the algorithm model still has room for improvement. Nowadays, the improved NSGA-II algorithm is being used in some 
construction fields to improve the population diversity and the progress of the selection process [13, 14], and NSGA-III algorithm was proposed in $2014[15,16]$. Therefore, it is the future research direction to further improve the accuracy of the multiobjective genetic algorithm and in finding optimal solutions for building energy efficiency under the influence of multiple complex factors. At the same time, we have to continue to improve and make the algorithm interface more understandable and easier to operate.

\section{Data Availability}

The data included in this paper are available without any restriction.

\section{Conflicts of Interest}

The authors declare that there are no conflicts of interest regarding the publication of this paper.

\section{References}

[1] J. P. Carvalho, M. Almeida, L. Bragança, and R. Mateus, "BIMbased energy analysis and sustainability assessment-application to Portuguese buildings," Buildings, vol. 11, no. 6, p. 246, 2021.

[2] Z. Pǔcko, D. Maǔcec, and N. Šuman, "Energy and cost analysis of building envelope components using BIM: a systematic approach," Energies, vol. 13, no. 10, p. 2643, 2020.

[3] S. Eleftheriadis, D. Mumovic, and P. Greening, "Life cycle energy efficiency in building structures: a review of current developments and future outlooks based on BIM capabilities," Renewable and Sustainable Energy Reviews, vol. 67, pp. 811825, 2017.

[4] R. Rasiulis, L. Ustinovichius, D. Migilinskas, J. Cepurnaite, and A. Virbickas, "Energy efficiency of a public building renovation and reconstruction using base model passive house and b technology," Engineering Structures and Technologies, vol. 7, no. 3, pp. 114-125, 2016.

[5] S. Beazley, E. Heffernan, and T. J. Mccarthy, "Enhancing energy efficiency in residential buildings through the use of BIM: the case for embedding parameters during design," Energy Procedia, vol. 121, pp. 57-64, 2017.

[6] K. Ding, J. O. Diaz, D. Bimberg, and C. Z. Ning, "Modulation bandwidth and energy efficiency of metallic cavity semiconductor nanolasers with inclusion of noise effects," Laser \& Photonics Reviews, vol. 9, no. 5, pp. 488-497, 2015.

[7] P. Moser, M. Volwahsen, G. Larisch, and J. A. Lott, "Maximizing the temperature insensitivity, energy efficiency, and bit rate of 980-nm VCSELs via small oxide-aperture diameters and photon lifetime tuning," Proceedings of SPIE - The International Society for Optical Engineering, vol. 9381, no. 12, p. 9381, 2015.

[8] P. Wolf, H. Li, A. Caliman, and A Mereuta, "Spectral efficiency and energy efficiency of pulse-amplitude modulation using waver-fusion VCSELs for optical interconnects," ACS Photonics, vol. 4, no. 8, pp. 2018-2024, 2017.

[9] M.-D. Yang, Y.-P. Chen, Y.-H. Lin, Y.-F. Ho, and J.-Y. Lin, "Multiobjective optimization using nondominated sorting genetic algorithm-II for allocation of energy conservation and renewable energy facilities in a campus," Energy and Buildings, vol. 122, pp. 120-130, 2016.
[10] Z. Q. Kang, Z.-X. Li, J. N. Dong, X. X. Zhou, and G. H. Feng, "Application of energy-saving technology of building equipments based on BIM," Building Energy Efficiency, vol. 45, no. 318, pp. 115-118, 2017.

[11] I. Njini and O. O. Ekabua, "Genetic algorithm based energy efficient optimization strategies in wireless sensor networks: a survey," Advances in Computer Science: an International Journal, vol. 3, no. 5, pp. 1-19, 2015.

[12] S. P Singh and S. C Sharma, "Genetic-algorithm-based energyefficient clustering (GAEEC) for homogenous wireless sensor networks," IETE Journal of Research, vol. 64, no. 5, pp. 648-659, 2017.

[13] W. Zhou, "Construction of genetic algorithm model for fitness program optimization of middle school students," in Proceedings of the 2021 International Conference on Bioinformatics and Intelligent Computing, pp. 189-195, Harbin, China, January 2021.

[14] Q.-X. Liang, "Optimization of longitudinal steel beam design of A continuous rigid bridge based on improved NSGA-II algorithm," Westem China Communications and Science Technology, vol. 167, no. 6, pp. 151-152, 2021.

[15] J. Zheng and J. Zhou, Multi-Objective Evolution Optimization, Science Press, Beijing, China, 2017.

[16] ZiX. Fan, S. Li, and J. W. Yu, "Multi-objective optimization of microgrid operation based on improved NSGA-III algorithm," Power System and Automation, vol. 43, no. 6, pp. 40-41, 2021. 